\title{
Characteristics of High Speed Steel/ductile Cast Iron Composite Roll Manufactured by Electroslag Remelting Cladding
}

\author{
Yulong $\mathrm{CAO},{ }^{1)}$ Yanwu DONG, ${ }^{2)}$ Zhouhua $\mathrm{JIANG}^{2{ }^{2 *}}{ }^{*}$ Guangqiang $\mathrm{LI}^{1)}$ and Zhengrong ZHAO \\ 1) The State Key Laboratory of Refractories and Metallurgy, Wuhan University of Science and Technology, Wuhan, 430081 \\ China. \\ 2) School of Metallurgy, Northeastern University, Shenyang, 110819 China.
}

(Received on September 27, 2020; accepted on March 18, 2021; J-STAGE Advance published date: June 2, 2021)

\begin{abstract}
In the present study, high-speed steel (HSS)/ductile cast iron (DCI) composite roll was manufactured by the electroslag remelting cladding (ESRC) technology. The compositional variation, grain size, microstructure, hardness, and tensile strength of the HSS layer and the bimetallic interface were investigated systematically. The obtained results illustrated that the chemical composition of the cladding layer (HSS) changed dramatically due to the surface melting of the roll core (DCl) and the mechanical mixing of the bimetallic liquids. The different solidification rates and chemical compositions in different regions of the HSS layer led to great variations of the grain size, the carbide content, and the hardness. In addition, a bimetallic transition zone (about $9.47 \mathrm{~mm}$ ) was generated between the HSS layer and the DCl core due to the elemental migration and diffusion between the bimetals. Carbides of different types, morphologies, sizes, and compositions had direct influences on interfacial properties.
\end{abstract}

KEY WORDS: high speed steel; composite roll; electroslag remelting; bimetallic transition zone; carbides.

\section{Introduction}

Roll is one of the key components of a rolling mill, and its performance directly affects the production efficiency of the mill, the surface quality of the rolled material, and the production cost of rolled steel. In recent years, rolling conditions are gradually becoming complicated and demanding in rolling plants due to the increasing volume of thin plates and high-strength steel plates, thus rolls with high hardness and excellent wear properties are required. In order to meet the above requirements, high-speed steel (HSS) rolls have been developed. ${ }^{1-3)}$

At present, in most cases, work rolls used for finishing stands of hot strip mills are generally composite rolls consisting of an outer shell made of centrifugally cast wear-resistant material (HSS) and a core made of ductile cast iron (DCI) or steel (centrifugally or statically cast).,5) Centrifugal casting has been widely used due to its advantages of high production efficiency, low cost and relatively mature technology, however, it also has the following disadvantages: (i) the density difference between carbides and the liquid steel in the HSS layer always results in severe segregation under the centrifugal force, ${ }^{6}$ (ii) coarse microstructures are always generated due to the limited casting

\footnotetext{
* Corresponding author: E-mail: jiangzh@smm.neu.edu.cn
}

cooling rate. Therefore, new methods have been developed for the fabrication of HSS composite rolls.

Electroslag remelting (ESR) is a popular process to produce clean steels with improved solidification structures. ${ }^{7}$ Exploiting the technological superiority of ESR and current supplying mold (CSM), ${ }^{8,9)}$ Jiang et al. ${ }^{10)}$ developed an innovative method of electroslag remelting cladding (ESRC) to manufacture composite rolls. Unlike centrifugal casting, in this method, the liquid metal of HSS layer is cladded around the solid roll core to form bimetallic composite billets and it can be not only refined by slag/metal reaction but also improved the solidification structure due to the rapid cooling. The HSS/DCI composite roll ${ }^{11)}$ has been manufactured by ESRC and some analyses about the bimetallic interface and DCI core have been carried out, in contrast, overall investigations on its HSS layer and integrated characteristics have rarely been published. Different from the HSS ingot fabricated by the conventional ESR technology, the HSS layer of an HSS/DCI composite roll presents peculiar microstructures and properties due to different heat transfer and interaction effects between HSS and DCI.

In the present study, the chemical composition, carbide characteristics (morphology, type, content), and hardness at different positions of the HSS layer and the bimetallic transition zone were analyzed. Furthermore, the technical features of ESRC and the effects of the DCI core on the HSS layer 
were integrally analyzed.

\section{Experimental Process and Methods}

In the ESRC process, the core part of a DCI ingot (diameter $=240 \mathrm{~mm}$ and length $=700 \mathrm{~mm}$ ) was placed vertically at the center of the water-cooled mold. A total of twenty rodlike HSS electrodes (diameter $=30 \mathrm{~mm}$ and length $=$ $1500 \mathrm{~mm}$ ) uniformly distributed around the DCI core were remelted in the high-temperature slag bath, and liquid droplets went through the slag bath and entered the gap between the mold and the roll core to form a molten metal bath of the HSS layer around the roll core (Fig. 1). The molten metal was then cooled by the adjacent water-cooled mold and roll core, then finally formed a metallurgical bonding between the HSS layer and the DCI core. The melting of the consumable electrodes and the cladding of the bimetals were controlled synchronously to obtain the target composite length. A detailed description of the preparation of composite rolls

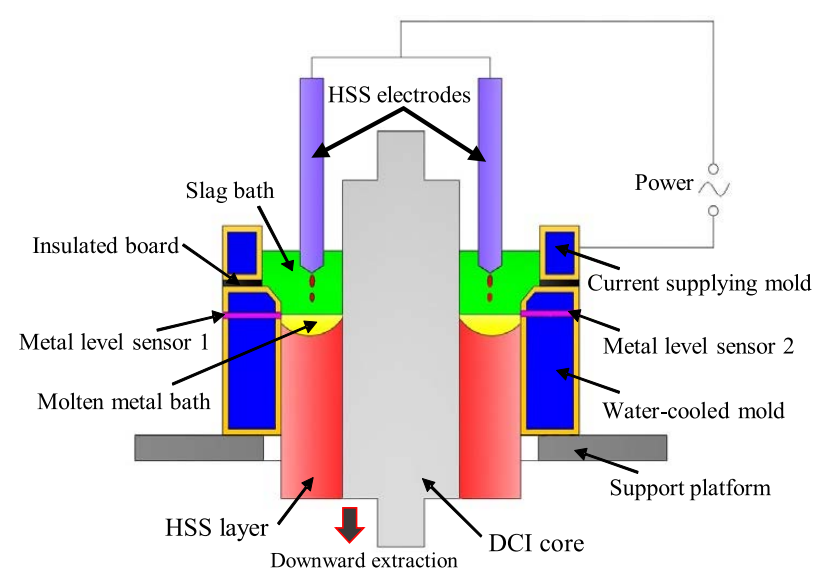

Fig. 1. Schematic diagram of the ESRC method. (Online version in color.)

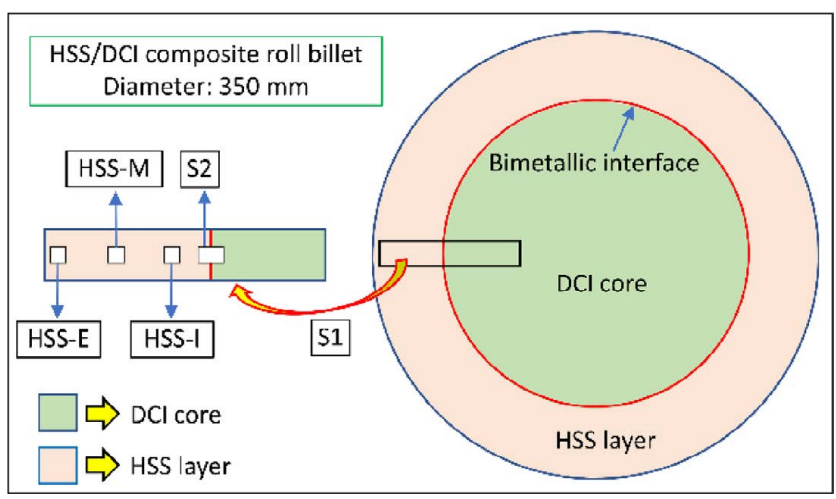

Fig. 2. Schematic diagram of the analytical specimens. (Online version in color.) by the ESRC method is presented in the literature. ${ }^{12)}$

An HSS/DCI composite roll with a diameter of $350 \mathrm{~mm}$ and a composite height of $264 \mathrm{~mm}$ was fabricated, and stress relief annealing was carried out at $750^{\circ} \mathrm{C}$ for six hours. Figure 2 presents the schematic diagram of the analytical specimens. The specimen marked as S1 (size $=110 \times 20 \mathrm{~mm})$ was taken from the cross-section of the composite billet, and it consisted of the HSS layer, the bimetallic interface, and the DCI core. After the macroanalysis and the spectral analysis, three specimens were taken from the outer edge, middle, and inner part of the HSS layer (marked as HSS-E, HSS-M, and HSS-I, respectively) for microstructural and hardness analyses. The specimen marked as S2 was used to analyze the carbide variation, microhardness, and elemental distribution in the bimetallic transition zone between HSS and DCI.

A direct reading spectrometer and a carbon-sulfur analyzer were used for macroscopic composition tests. An optical microscope was used to characterize the carbide morphology, and quantitative analyses were performed on the area percentage of carbides based on multiple pictures per specimen. Before the quantitative analysis, the specimens were ground, polished and then etched by $4 \%$ nital. The carbide composition was determined by an energy dispersive X-ray spectrometer (EDS) attached to the scanning electron microscope (SEM). The quantitative analysis of elemental distributions at different regions of the bimetallic transition zone was carried out by electron probe microanalysis (EPMA). A Vickers hardness tester was employed for macrohardness and microhardness analyses under loads of $49.03 \mathrm{~N}$ and $4.903 \mathrm{~N}$, respectively. The microhardness tests in the bimetallic transition zone were conducted with the distance of $500 \mu \mathrm{m}$ between two adjacent points.

\section{Results and Discussions}

\subsection{Chemical Composition of HSS Layer}

Figure 3 displays the macroscopic features of the S1 specimen after etching by $4 \%$ nital. It is evident that an obvious bimetallic transition zone is located between the HSS layer and the DCI core. During the ESRC process, the Joule heat generated due to the electric current passing through the slag bath was the heat source of the entire composite system, and it remelted the HSS electrodes to form a molten metal bath of the HSS layer and also preheated the DCI core to a high-temperature state ${ }^{13)}$ to promote metallurgical bonding between the bimetals. As the DCI core had a lower melting temperature than the HSS electrodes, a certain degree of surface melting was inevitable to realize the liquid/solid bonding and obtain a bonding interface without defects between the bimetals. With the surface melting of the DCI core, these two types of liquid metals were mixed

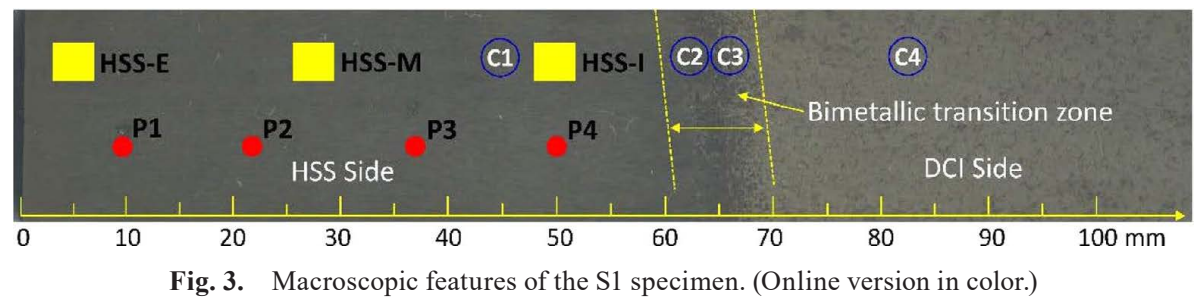


mechanically, leading to a chemical composition variation of the HSS layer. Moreover, the shell thickness of the HSS layer increased with the decreasing diameter of the DCI core. For the chemical composition analysis, four points on the HSS layer (marked as P1, P2, P3, P4) and four points across the bimetallic transition zone (marked as $\mathrm{C} 1, \mathrm{C} 2, \mathrm{C} 3$, C4) were selected (Fig. 3).

The analysis results for points P1 to P4 on the HSS layer obtained by direct reading spectrometry are presented in Table 1. Compared with the initial composition of HSS electrodes, as a whole, an increase in the contents of $\mathrm{C}$, $\mathrm{Si}, \mathrm{Mn}, \mathrm{Ni}$ and a decrease in the contents of $\mathrm{W}, \mathrm{Mo}, \mathrm{Cr}, \mathrm{V}$ occurred due to the compositional difference between the bimetals (as HSS with higher $\mathrm{W}, \mathrm{Mo}, \mathrm{Cr}, \mathrm{V}$ and DCI with higher $\mathrm{C}, \mathrm{Si}, \mathrm{Mn}, \mathrm{Ni}$ ). In comparison to points P1, P2, P3, point $\mathrm{P} 4$ had higher $\mathrm{C}, \mathrm{Si}, \mathrm{Mn}, \mathrm{Ni}$ elements and lower $\mathrm{W}$, $\mathrm{Mo}, \mathrm{Cr}, \mathrm{V}$ contents because it was closest to the ductile iron side with strong elemental migration and diffusion behavior. Moreover, between the area between P1 and P3, the content of each element had a certain fluctuation. The temperature of the contact area between the roll core and the slag bath was higher than that of the contact area between the roll core and the cladding layer, ${ }^{10)}$ therefore, the melting zone of the roll core surface was formed in the contact part with the slag bath and slid down from the roll core to have a mechanical mixing with the liquid metal of the HSS layer due to the density difference between the DCI core and the liquid slag. Except for the possibility of uneven mixing, the following two aspects might also be the reasons for the compositional fluctuation from points $\mathrm{P} 1$ to $\mathrm{P} 3$ in the HSS layer. First, in the ESRC system, the water-cooled mold and the solid DCI core with a weaker cooling capacity act as a casting mold during the solidification process of the molten HSS metal; therefore, the final solidification zone (FSZ) in the HSS layer was always located at its middle zone near the DCI core in the radial direction. Second, W, Mo, Cr, and V in the HSS layer were positive segregation elements; thus, they were always enriched in the macroscopic FSZ and the microscopic grain boundary. As the position of $\mathrm{P} 3$ in Fig. 3 is likely to be the FSZ of the HSS layer, the contents of W, Mo, and V were higher than those of P1 and P2 (Table 1).

An equation of stoichiometric carbon equivalent $(\mathrm{SCE})^{14)}$ was used to calculate the quantity of carbon that formed carbides and the carbon solution in the matrix.

$$
\mathrm{SCE}=0.06 \mathrm{Cr}+0.033 \mathrm{~W}+0.06 \mathrm{Mo}+0.2 \mathrm{~V}(\mathrm{wt} \%) \ldots
$$

Carbon content (in the matrix $)=\% \mathrm{C}($ base metal $)-\mathrm{SCE}$

The carbon content in the matrix at points P1, P2, P3, and $\mathrm{P} 4$ were calculated as $0.41 \%, 0.38 \%, 40 \%$, and $0.51 \%$, respectively. It determines the transformation characteristics of the matrix and a value greater than $0.40 \%$ seriously harms the fracture toughness due to the increased amount of plate martensite. ${ }^{4)}$ In this work, due to the stress relief annealing heat treatment, the as-cast matrix structures of martensite and retained austenite are decomposed into different types of carbides as well as the ferrite with low hardness. The Rockwell hardness values at these four points were 38.3 HRC, 39.3 HRC, 40.2 HRC, and 42.6 HRC, respectively, which are closely related to the type, size, quantity, and distribution of carbides and the matrix hardness (usually proportional to the carbon content in the matrix).

\subsection{Microstructure of HSS Layer}

Figure 4 exhibits the microstructures of HSS-E, HSS-M, and HSS-I specimens shown in Fig. 2. It is clear they are consisted of dendrites of primary austenite and networks of eutectic carbides distributed in interdendritic regions. It is noticeable that as compared to HSS-M, dendrites were more refined, and networks of eutectic carbides were distributed more homogeneously in HSS-E and HSS-I specimens. In these specimens, two types of carbides were mainly formed, granular or blocky MC carbides and rodlike or lamellar $\mathrm{M}_{2} \mathrm{C}$ carbides. $\gamma+\mathrm{M}_{6} \mathrm{C}$ eutectic was rarely formed as the solidification normally occurred under non-equilibrium conditions, and the formation of metastable $\gamma+\mathrm{M}_{2} \mathrm{C}$ eutectic was favored due to the high cooling rate and low local solidification time for ESR ingots. ${ }^{15)}$

The cooling rate is an important factor affecting the type and morphology of eutectic carbides. ${ }^{16,17)}$ A larger cooling rate is beneficial to the formation of $\mathrm{M}_{2} \mathrm{C}$ carbides and changes the morphology of $\mathrm{M}_{2} \mathrm{C}$ carbides from lamellar to rodlike. ${ }^{18)}$ The cooling rate is the product of temperature gradient $(G)$ and growth rate $(V)$. As the cooling rate $\left(G^{*} V\right)$ in the edge zone was larger than those in the middle and inner zones, a larger degree of supercooling and a higher number of fine columnar dendrites were obtained in the HSS-E specimen, whereas eutectic carbides in the middle and inner zones could grow for a sufficient time during solidification and formed thicker and shorter rodlike $\mathrm{M}_{2} \mathrm{C}$ carbides, a good agreement between the above results and the reports by $\mathrm{Yin}^{19)}$ has been obtained. As the affinity between $\mathrm{V}$ and $\mathrm{C}$ is stronger than that between $\mathrm{W}, \mathrm{Mo}, \mathrm{Cr}$, and $\mathrm{C}$, the increasing content of $\mathrm{C}$ and a certain content of $\mathrm{V}$ in the HSS-I specimen led to the formation of more granular MC carbides.

The statistical analysis results for the area percentage of eutectic carbides, average grain-size grade value, and Vickers hardness of the three specimens on the HSS layer

Table 1. Chemical compositions of initial HSS electrodes and different research points.

\begin{tabular}{cccccccccccc}
\hline Specimen & $\mathrm{C}$ & $\mathrm{Si}$ & $\mathrm{Mn}$ & $\mathrm{P}$ & $\mathrm{S}$ & $\mathrm{Cr}$ & $\mathrm{Mo}$ & $\mathrm{V}$ & $\mathrm{W}$ & $\mathrm{Ni}$ & $\mathrm{Fe}$ \\
\hline Initial HSS & 0.89 & 0.37 & 0.32 & 0.025 & 0.004 & 4.03 & 4.76 & 1.85 & 6.19 & 0.19 & Bal. \\
P1 & 1.24 & 0.59 & 0.36 & 0.026 & 0.002 & 3.47 & 3.54 & 1.29 & 4.42 & 0.75 & Bal. \\
P2 & 1.21 & 0.60 & 0.35 & 0.024 & 0.001 & 3.30 & 3.61 & 1.37 & 4.41 & 0.74 & Bal. \\
P3 & 1.26 & 0.61 & 0.36 & 0.027 & 0.002 & 3.36 & 3.86 & 1.39 & 4.48 & 0.76 & Bal. \\
P4 & 1.31 & 0.67 & 0.39 & 0.028 & 0.001 & 3.29 & 3.53 & 1.26 & 4.10 & 0.89 & Bal. \\
\hline
\end{tabular}



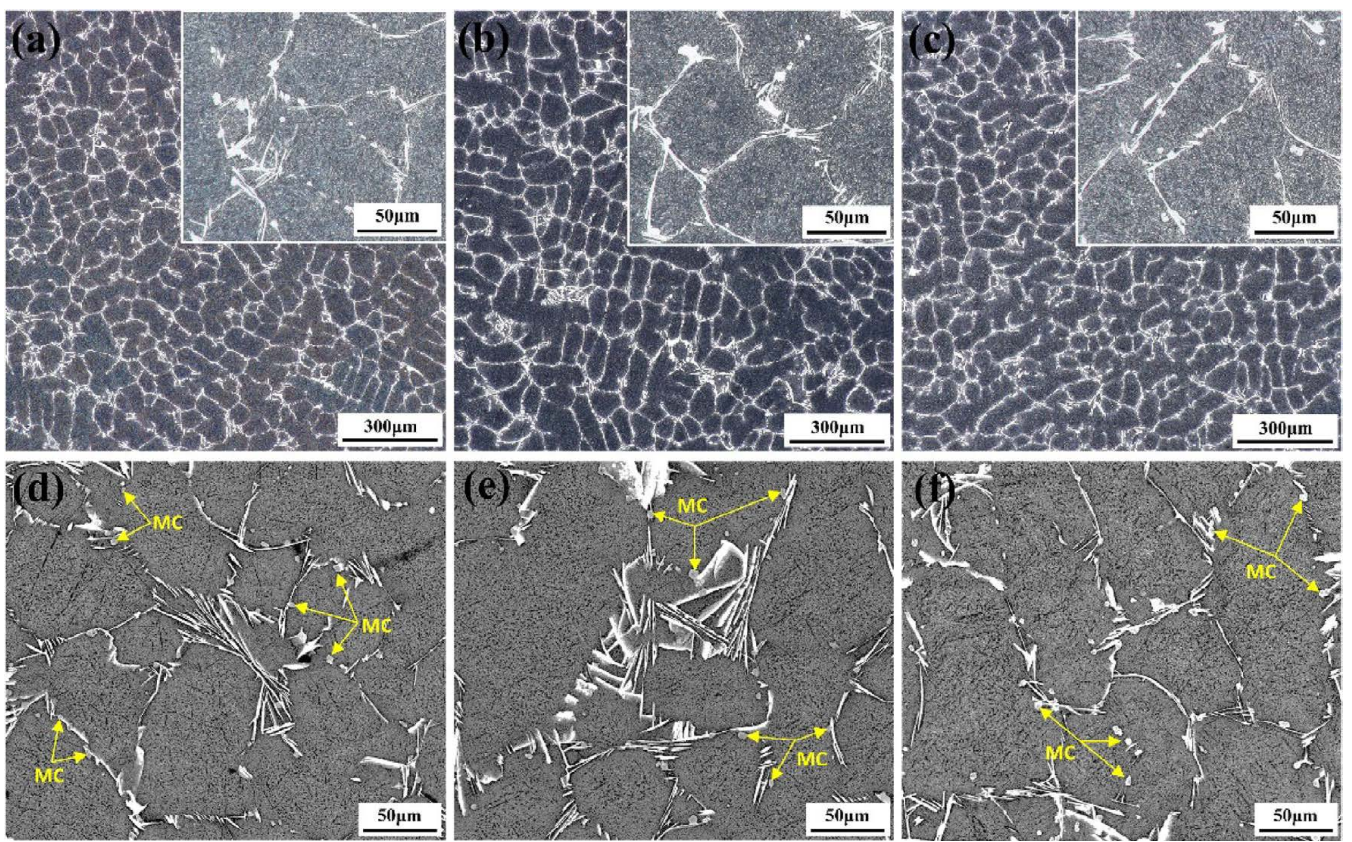

Fig. 4. Microstructures of (a) HSS-E, (b) HSS-M, (c) HSS-I specimens, (d-f) Scanning analysis of the HSS layer (Online version in color.)

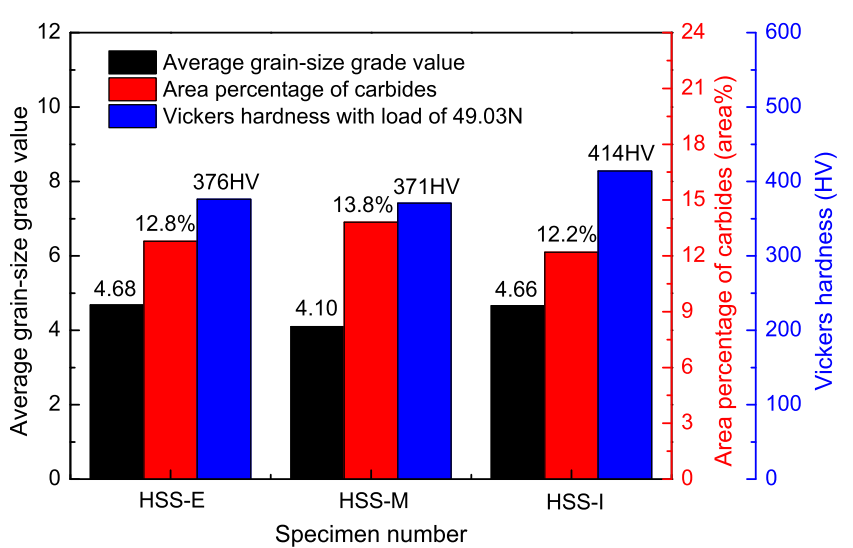

Fig. 5. Parameter values of different specimens in the HSS layer. (Online version in color.)

are depicted in Fig. 5. Here, the average grain-size grade value is measured by the circular cutoff point method. ${ }^{20)}$ As larger grain-size grade value represents finer grain, the finest and coarsest grains were obtained at the edge and middle zones of the HSS layer, respectively which are closely related to the cooling rate. The carbide contents in HSS-E and HSS-I specimens were similar and lower than those in HSS-M specimen. The Vickers hardness values of HSS-E and HSS-M specimens were the almost same and lower than that of the HSS-I specimen.

According to the above results of chemical composition and average grain-size grade value, it is discernible that the final solidification zone of the HSS layer was most likely to occur around HSS-M and point P3. Hence, the contents of $\mathrm{W}, \mathrm{Mo}, \mathrm{Cr}, \mathrm{V}$ in HSS-M were higher than those of HSS-E and HSS-I. As the carbide content in steel is inversely proportional to the cooling rate ${ }^{21)}$ and directly proportional to the contents of carbon and carbide-forming elements, ${ }^{4,22}$ ) the largest area percentage of carbides was obtained in the HSS-M specimen. In the HSS-I specimen, more MC car- bides with high hardness were found due to the increasing carbon content; thus, it had the highest Vickers hardness.

Figure 6 displays the surface scanning analysis result of carbides in the HSS-E specimen, the brighter the color, the more content the element. It is clear that granular MC carbides (gray) were mostly enriched with $\mathrm{V}$ and a certain amount of $\mathrm{W}$ and $\mathrm{Mo}$, whereas $\mathrm{M}_{2} \mathrm{C}$ carbides (white) were mostly enriched with $\mathrm{Mo}, \mathrm{W}$, and a certain amount of $\mathrm{V}$. The two types of carbides are all poor in $\mathrm{Cr}$ and $\mathrm{Fe}$ because these types of carbides tend to preferably contain elements other than $\mathrm{Fe}$ and $\mathrm{Cr}$, typically $\mathrm{V}$ in case of $\mathrm{MC}$ and $\mathrm{Mo}$ in case of $\mathrm{M}_{2} \mathrm{C}$.

$\mathrm{M}_{2} \mathrm{C}$ carbides were generated by the eutectic reaction, $\mathrm{L} \rightarrow \mathrm{M}_{2} \mathrm{C}+\gamma$, the process of which is accompanied by the element diffusion in the liquid steel. ${ }^{23)}$ With the increasing cooling rate, the elemental diffusion became insufficient; thus, the concentrations of alloying elements in $\mathrm{M}_{2} \mathrm{C}$ decreased, and those in the matrix increased. In addition, the morphology of $\mathrm{M}_{2} \mathrm{C}$ was transformed from lamellar to rodlike. $\mathrm{M}_{2} \mathrm{C}$ eutectic carbides were metastable and decomposed into finer $\mathrm{M}_{6} \mathrm{C}$ and $\mathrm{MC}$ carbides at high temperatures. ${ }^{24-26)}$ In comparison to lamellar carbides, rodlike $\mathrm{M}_{2} \mathrm{C}$ was less stable and decomposed faster at high temperatures, accelerating the separation and spheroidization of carbides even after hot deformation. The formation of rodlike $\mathrm{M}_{2} \mathrm{C}$ in cast ingots promoted the homogeneous distribution and refinement of carbides in the final products and favored the improvement of mechanical properties (hardness, wear resistance, impact toughness) of HSS. ${ }^{27)}$

\subsection{Analysis of Bimetallic Transition Zone}

A specimen marked as S2 was taken from the S1 specimen (Fig. 2) and used to carry out a systematic analysis of the bimetallic transition zone between the HSS layer and the DCI core. The microstructural change in this zone is displayed in Fig. 7. From the HSS layer to the DCI core, five typical microstructures (taken from $\mathrm{K} 1$ to $\mathrm{K} 5$ areas) 

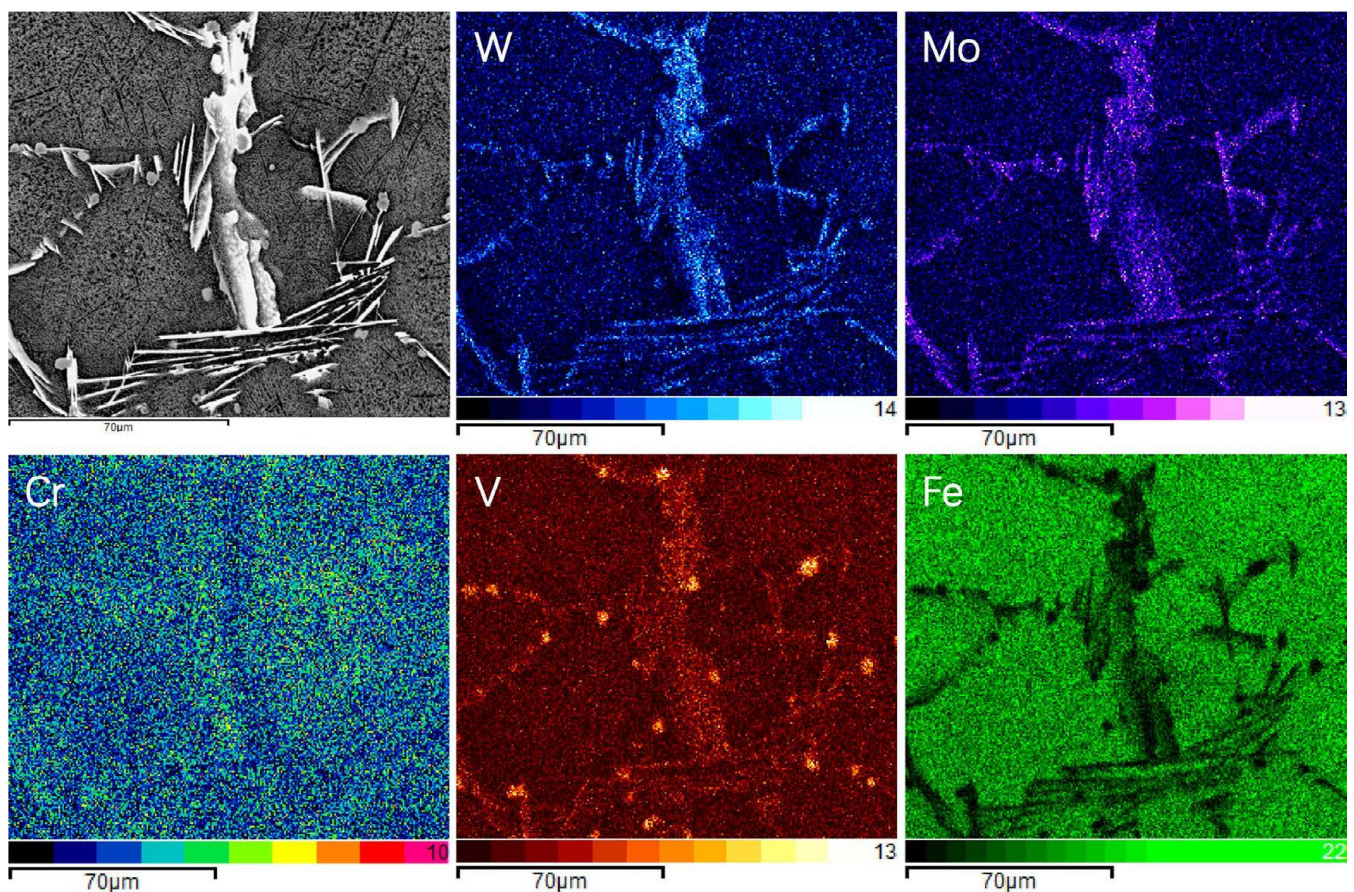

Fig. 6. Surface scanning analysis of carbides in the HSS-E specimen. (Online version in color.)

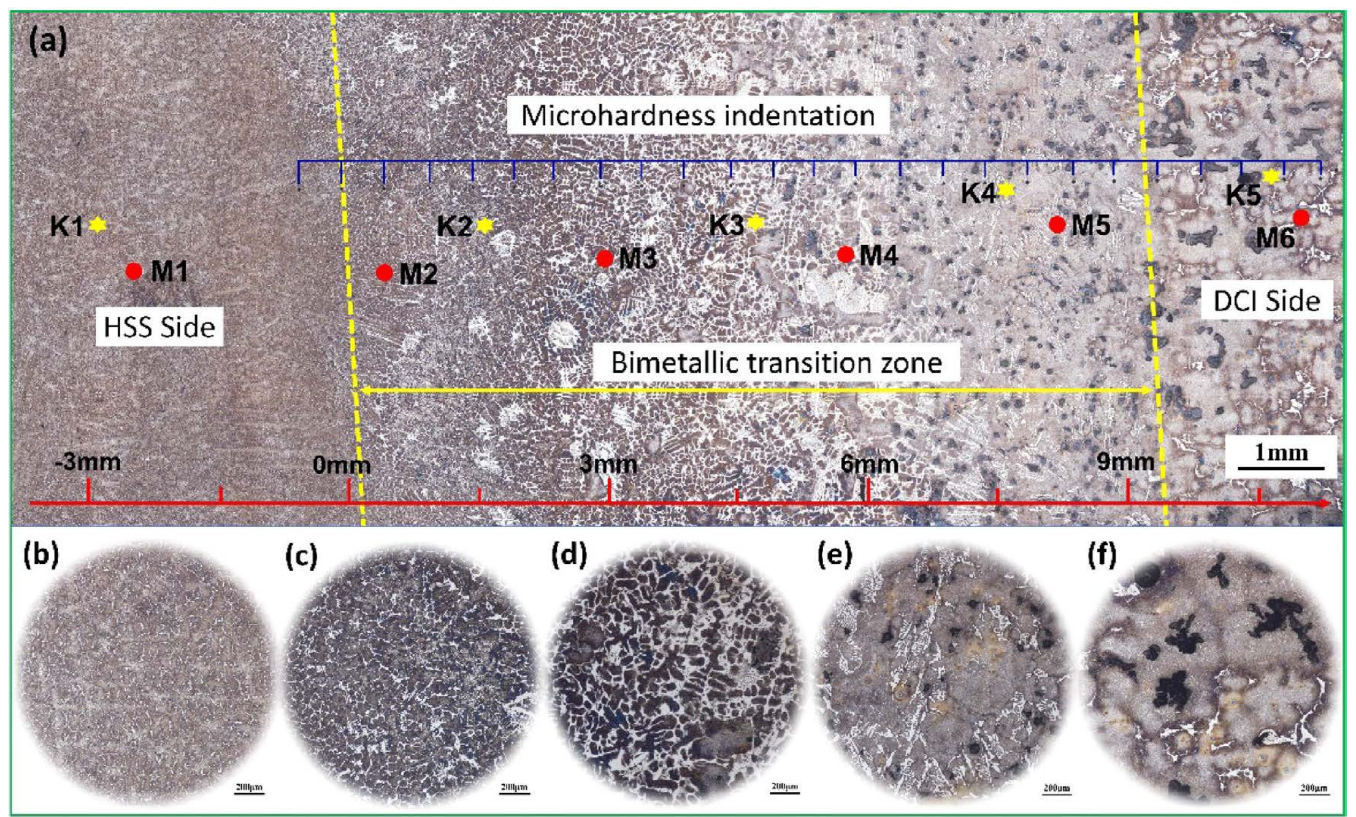

Fig. 7. Microstructural change in the bimetallic transition zone between the HSS layer and the DCI core. (Online version in color.)

were sequentially generated (Figs. 7(b)-7(f)) respectively. Figure 7(b) indicates the formation of fine granular and rodlike carbides without graphite in the HSS side; however, in the direction to the DCI side (Figs. 7(c) to 7(e)), a large number of coarse (plates) carbides were formed at first and then disappeared to form a clear ledeburite eutectic structure with a certain amount of graphite particles. In the DCI side, large graphite particles with poor sphericity and a flowerlike morphology were non-uniformly distributed, and a few dispersed network carbides were formed (Fig. 7(f)). The microstructural change across the bimetallic transition zone can be attributed to the elemental migration and diffusion between the bimetals. Therefore, four points marked as $\mathrm{C}$, $\mathrm{C} 2, \mathrm{C} 3, \mathrm{C} 4$ in Fig. 3 were used to measure the macroscopic carbon change by a carbon sulfur analyzer, and six points (M1 to M6) shown in Fig. 7 were used to detect the microscopic composition change in the matrix from the HSS layer to the DCI core by EPMA. In this position, the boundary of HSS side and bimetallic transition zone is defined as the zero point in the horizontal axis.

The carbon content gradually increased from $1.34 \%$, $1.91 \%, 2.46 \%$ to $2.57 \%$ from points $\mathrm{C} 1$ to $\mathrm{C} 4$. Figure 8 illustrates the microscopic composition analysis results, and a decreasing trend of carbide-forming elements and an increasing trend of $\mathrm{Si}, \mathrm{Mn}$, and Ni elements occurred from M1 to M6, and it can be ascribed to the local macroscopic composition (high $\mathrm{W}, \mathrm{Mo}, \mathrm{Cr}, \mathrm{V}$ in the HSS layer and high $\mathrm{Si}, \mathrm{Mn}, \mathrm{Ni}$ in the DCI core) and nearby carbides. There- 

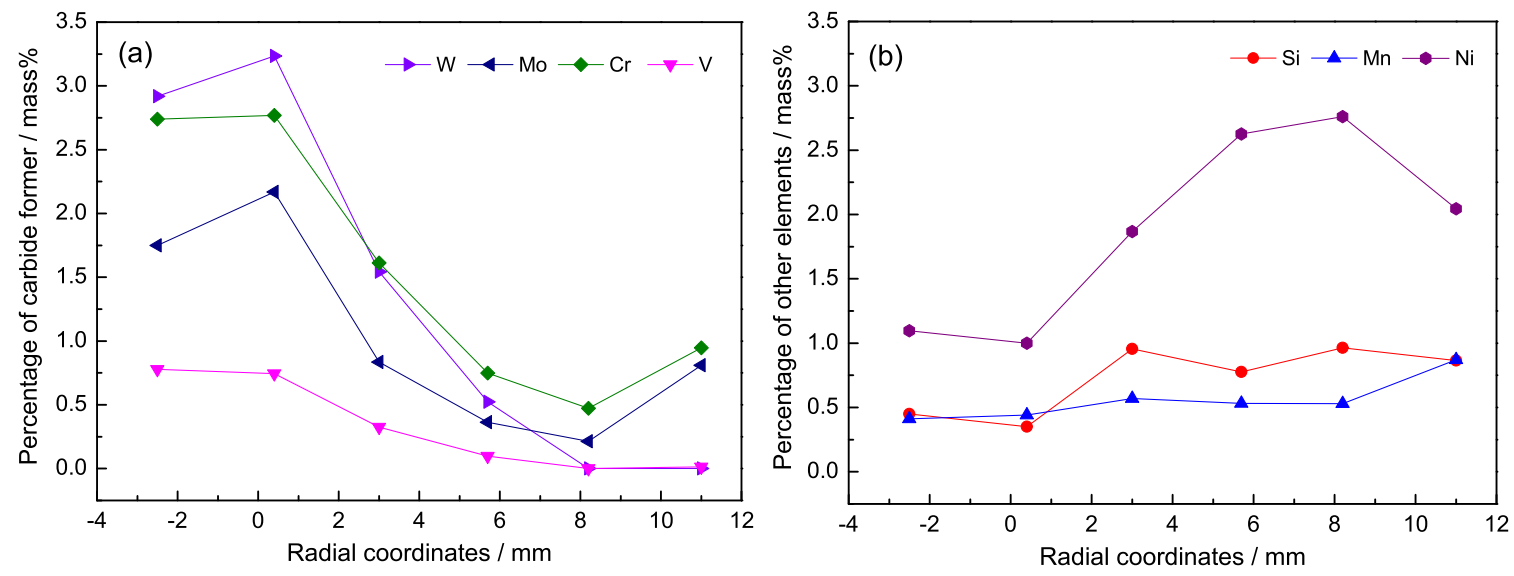

Fig. 8. Distributions of (a) carbide-forming elements and (b) other elements in the bimetallic transition zone (The zero point in the horizontal axis corresponds to that of Fig. 7). (Online version in color.)
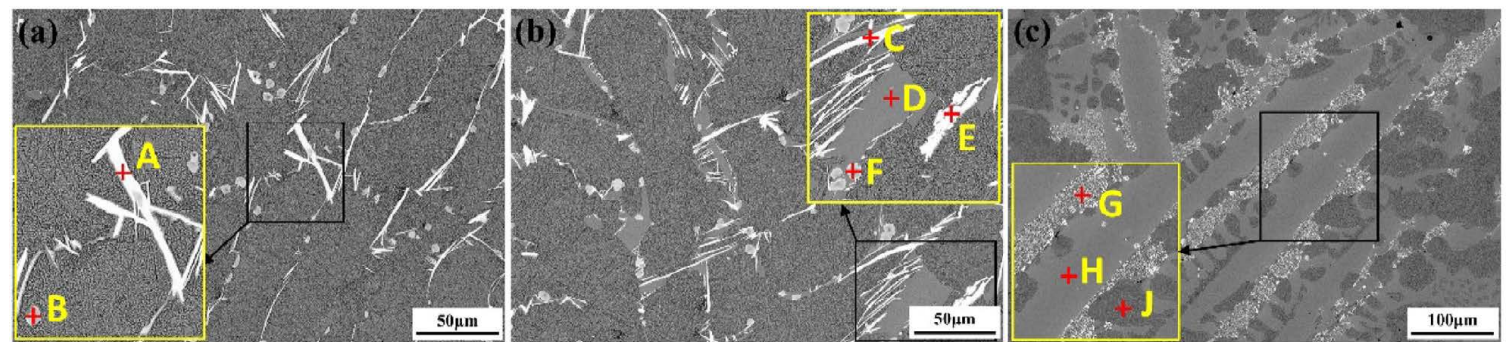

Fig. 9. Point scanning analysis of three typical carbides in the bimetallic transition zone. (Online version in color.)
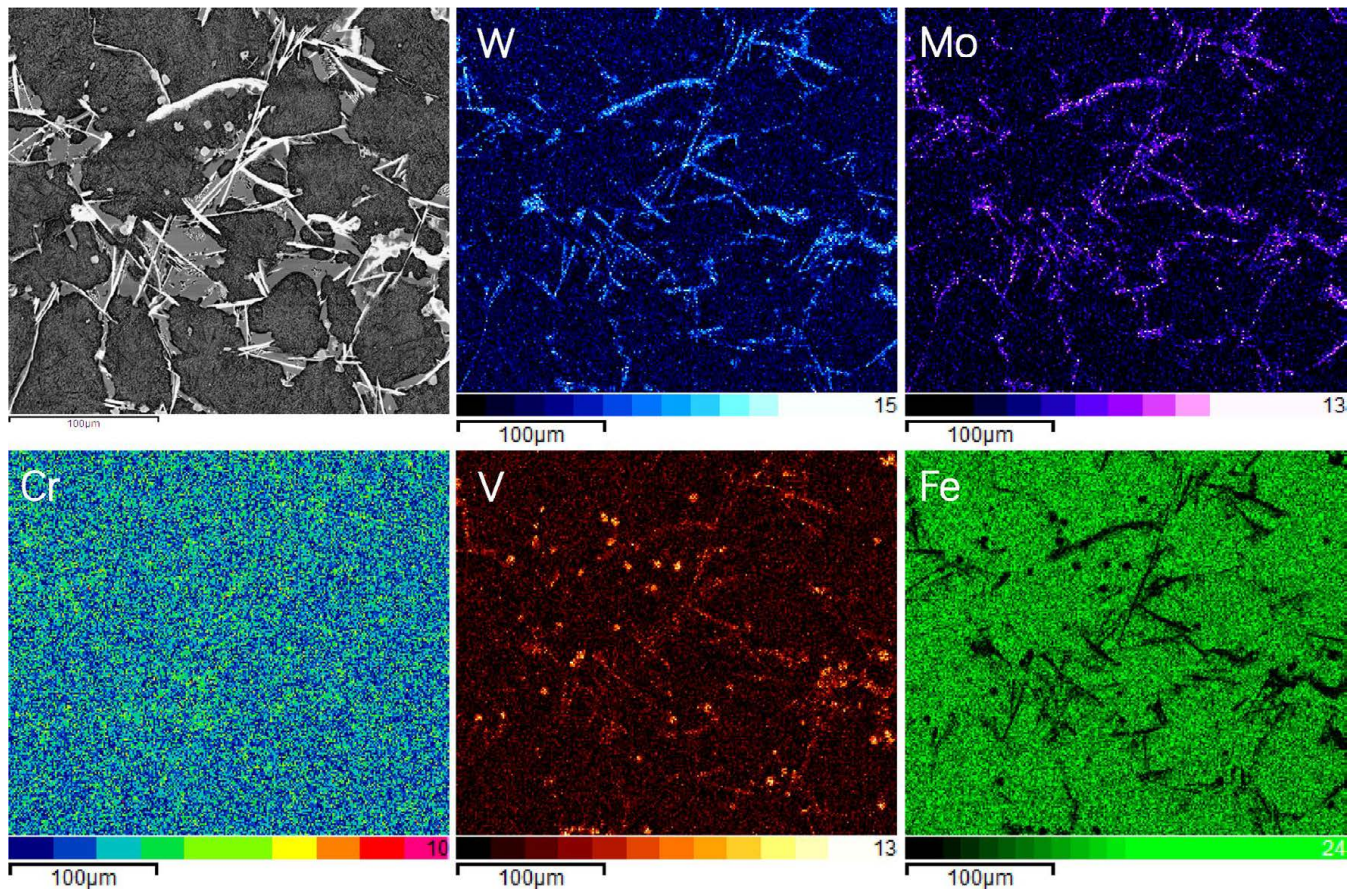

Fig. 10. Surface scanning analysis of compound carbides in the bimetallic transition zone. (Online version in color.)

fore, the contents of $\mathrm{C}, \mathrm{Si}, \mathrm{Mn}$, and Ni increased gradually in the bimetallic transition zone from the HSS layer to the DCI core, whereas those of $\mathrm{W}, \mathrm{Mo}, \mathrm{Cr}$, and $\mathrm{V}$ showed the opposite trend.

In the present study, three typical carbides (Figs. 7(b), $7(\mathrm{c}), 7(\mathrm{e}))$ were selected to have a clear understanding of their compositional characteristics by point (Fig. 9) and surface scanning analyses (Fig. 10). The atomic percentages of carbide-forming elements at different points are presented in Table 2. In Fig. 9(a), rodlike $\mathrm{M}_{2} \mathrm{C}$ (point $\mathrm{A}$ ) and granular $\mathrm{MC}$ (point $\mathrm{B}$ ) are mainly noticeable, the compositions of $\mathrm{MC}$ and $\mathrm{M}_{2} \mathrm{C}$ carbides are similar to that of M2 HSS steel. ${ }^{22}$ ) Carbides in Fig. 9(c) are mainly fishbone-type ledeburite. At point $\mathrm{G}$, dispersed carbides consisting of Fe and a certain content of $\mathrm{W}$ and Mo were generated around $\mathrm{M}_{3} \mathrm{C}$ carbides (point $\mathrm{H}$ ). At point $\mathrm{J}$ in the matrix, fewer $\mathrm{W}, \mathrm{Mo}, \mathrm{Cr}$, and 
Table 2. Chemical compositions of different carbides (atomic\%) (Points A-J are indicated in Fig. 9).

\begin{tabular}{crrrrrc}
\hline Point & \multicolumn{1}{c}{ W } & \multicolumn{1}{c}{ Mo } & \multicolumn{1}{c}{ Cr } & \multicolumn{1}{c}{ V } & \multicolumn{1}{c}{ Fe } & Type \\
\hline A & 21.37 & 29.73 & 10.57 & 27.82 & 10.52 & $\mathrm{M}_{2} \mathrm{C}$ \\
B & 13.52 & 12.56 & 5.20 & 63.70 & 5.02 & $\mathrm{MC}$ \\
C & 22.99 & 38.02 & 13.37 & 12.33 & 13.29 & $\mathrm{M}_{2} \mathrm{C}$ \\
D & 1.75 & 4.08 & 13.43 & 2.58 & 78.16 & $\mathrm{M}_{3} \mathrm{C}$ \\
E & 23.61 & 39.20 & 10.88 & 9.50 & 16.82 & $\mathrm{M}_{2} \mathrm{C}$ \\
F & 14.57 & 14.43 & 5.63 & 60.67 & 4.70 & $\mathrm{MC}$ \\
G & 3.83 & 7.07 & 1.84 & 0.90 & 86.36 & - \\
H & 0.80 & 1.25 & 6.21 & 2.30 & 89.44 & $\mathrm{M}_{3} \mathrm{C}$ \\
J & 0.62 & 0.83 & 1.43 & 0.33 & 96.78 & Matrix \\
\hline
\end{tabular}

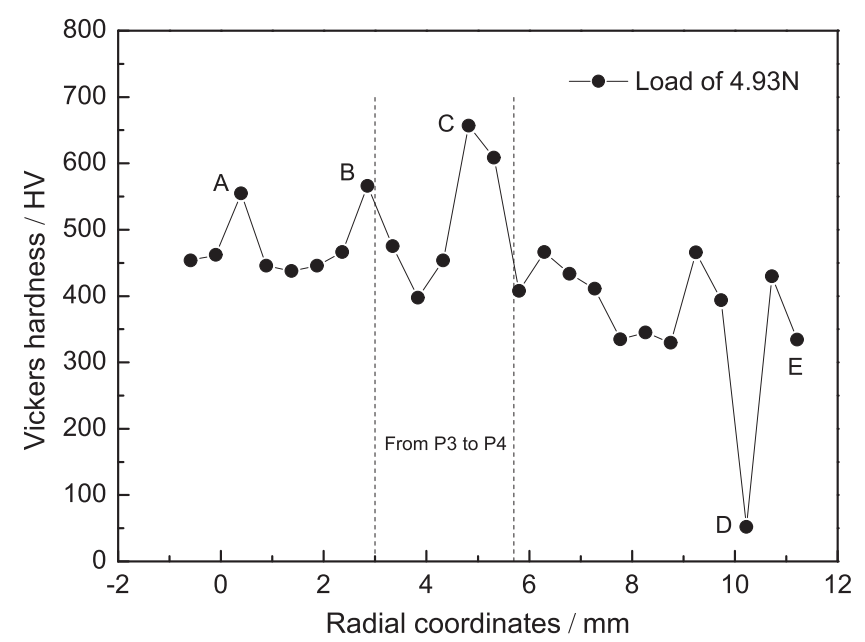

Fig. 11. Microhardness distribution in the bimetallic transition zone (The zero point in the horizontal axis corresponds to that of Fig. 7).

$\mathrm{V}$ dissolved. Unlike the carbides in the areas near HSS side or DCI side, those near the central zone of the bimetallic transition zone were compound ones (Fig. 9(b)), and rodlike $\mathrm{M}_{2} \mathrm{C}$ (point $\mathrm{C}$ ) or granular $\mathrm{MC}$ (point $\mathrm{F}$ ) carbides were always generated on the substrate of large plate $\mathrm{M}_{3} \mathrm{C}$ carbides (point $\mathrm{D}$ ). In $\mathrm{M}_{3} \mathrm{C}$ carbides, it mainly included $\mathrm{Fe}$ and $\mathrm{C}$, Fe was replaced by $\mathrm{Cr}, \mathrm{W}, \mathrm{Mo}$, and $\mathrm{V}$ to some extent to form alloying cementite, among these elements, $\mathrm{Cr}$ was the preferred element for the replacement of Fe. As the density $\left(\mathrm{g} / \mathrm{cm}^{3}\right)$ difference among $\mathrm{W}$ (19.1), Mo (10.2), Cr (7.20), V (5.8), and $\mathrm{Fe}$ (7.86) is very high, $\mathrm{M}_{2} \mathrm{C}$ carbides were brighter than $\mathrm{MC}$ and $\mathrm{M}_{3} \mathrm{C}$ ones under the backscattering mode of SEM due to the high contents of heavy elements (W, Mo). From Fig. 10, the contents of $\mathrm{W}, \mathrm{Mo}$, and $\mathrm{V}$ in plate $\mathrm{M}_{3} \mathrm{C}$ carbides were similar to those in the matrix. Bright rodlike $\mathrm{M}_{2} \mathrm{C}$ and granular $\mathrm{MC}$ carbides were rich in $\mathrm{W}, \mathrm{Mo}$, and $\mathrm{V}$, and poor in $\mathrm{Fe}$.

Figure 11 displays the microhardness distributions in the bimetallic transition zone (test points are marked in Fig. 7). By and large, a gradual decrease in the values occurred from the HSS layer to the DCI side because of the decreasing trend of alloying elements in the matrix (Fig. 8(a)). During the tests, the first point and the distance between two adjacent points were selected first, and then the other test points were automatically determined along the set direction. It

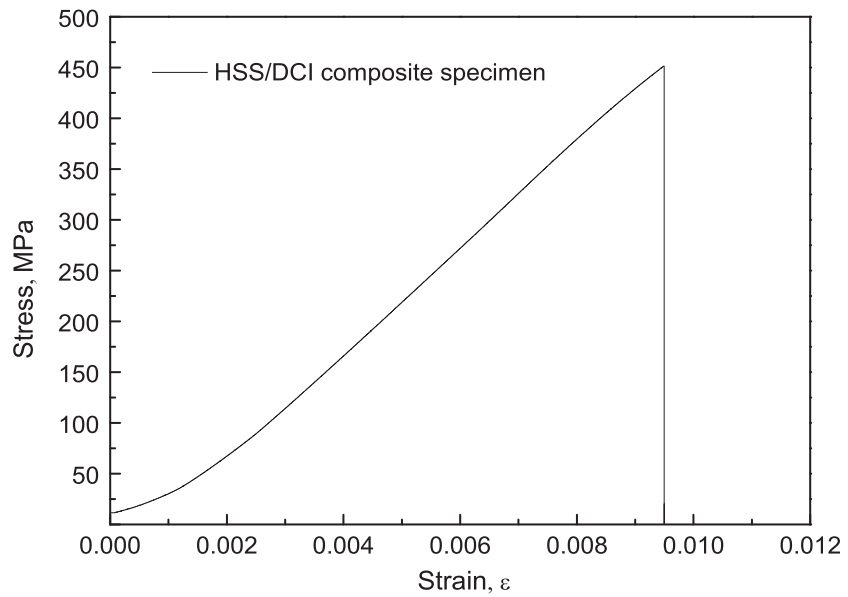

Fig. 12. Stress versus strain curve for the HSS/DCI composite specimen.

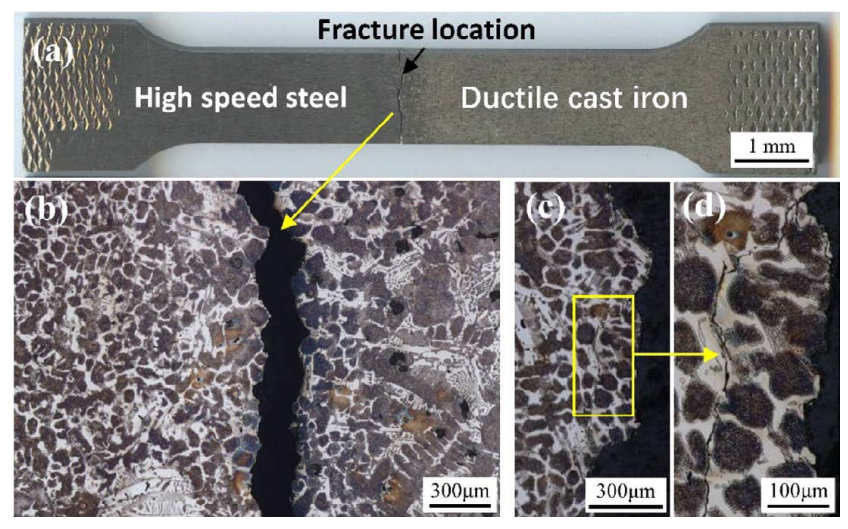

Fig. 13. Macromorphology and microstructure of the tensile fracture. (Online version in color.)

is worth noting that when the durometer head fell on the hard phase (carbides) or the soft phase (graphite particles), the significantly higher or lower hardness values occurred (points A, B, C, D in Fig. 11). In addition, higher hardness values were obtained at the radial coordinates between 3 $\mathrm{mm}$ and $6 \mathrm{~mm}$ corresponding to the area between M3 and M4 in Fig. 7.

In order to evaluate the bonding quality of the bimetallic transition zone, tensile tests for the specimens including the transition zone were carried out with a tensile speed of $0.5 \mathrm{~mm} \cdot \mathrm{min}^{-1}$, here, the strain is the ratio of the total tensile displacement to the original length of the specimen before the tensile tests. It is noticeable that a proportional stress-strain line was generated until the fracture occurred, and tensile strength of $452 \mathrm{MPa}$ was measured with neither obvious plastic deformation nor yielding phenomenon as shown in Fig. 12. The macroscopic morphology of the tensile fracture in Fig. 13 expresses that the reduction of area and elongation were very low, revealing the occurrence of brittle fracture.

Figure 13(a) reveals that the tensile fracture of the composite specimen occurred in the bimetallic transition zone, which indicates that here is the weak zone of the bimetallic composite roll. Judging from Figs. 13(b) to 13(d), we can conclude that the formation and propagation of microcracks along the carbide/matrix interface or carbides themselves were the main reasons for the fracture under the tensile load. 
As carbides were harder than the matrix, they were more difficult to deform under the tensile load; hence, a stress concentration occurred between carbides and the matrix and eventually led to the fracture. The fracture appeared in the area where the dissolution and disappearance of graphite particles occurred; thus, a large number of coarse network carbides were generated in this position (Fig. 13(b)). This position corresponded to the area between points M3 and M4 in Fig. 7 and had the maximum hardness value (Fig. 11). Therefore, it can be inferred that a uniform distribution of fine carbides is necessary to improve the bonding quality between the HSS layer and the DCI core.

\section{Conclusions}

An ESRC process consisting of ESR and CSM technologies was adopted to fabricate the HSS/DCI composite roll, and systematic analyses of the HSS layer and the bimetallic transition zone were carried out. The main observations of the present experiments are depicted below.

(1) The fusion of the DCI core surface occurred due to the high temperature in the slag bath and the low melting temperature of DCI, changing the chemical compositions of the HSS layer and the bimetallic transition zone generated between the HSS layer and the DCI core. As the contents of $\mathrm{C}, \mathrm{Si}, \mathrm{Mn}$, and Ni in the DCI core were higher than those in the HSS electrodes, these elements increased in the HSS layer, whereas carbide-forming elements (W, Mo, Cr, and $\mathrm{V})$ decreased due to the interaction of the bimetals.

(2) During the ESRC process, the molten metal of the HSS layer was cooled simultaneously by the adjacent watercooled mold and the solid DCI core. As the former had a larger cooling capacity than the latter, the final solidification zone of the HSS layer was located near the DCI core in the radial direction instead of its middle zone. Hence, the coarser grains and more carbides were obtained in HSS-M specimen in compared with those in the HSS-E and HSS-I specimens. All three specimens were mainly composed of rodlike $\mathrm{M}_{2} \mathrm{C}$ and granular $\mathrm{MC}$ carbides with a minor difference in morphological and elemental distribution features.

(3) In the bimetallic transition zone, a great difference in the morphologies of graphite and carbides was noticed, the closer to the DCI side, the lower the amount of $\mathrm{M}_{2} \mathrm{C}$ and $\mathrm{MC}$ carbides with a fine morphology and the higher the content of compound carbides (with the substrate of large plate $\mathrm{M}_{3} \mathrm{C}$ carbides) or ledeburite with a coarse morphology due to the increase of $\mathrm{C}$ and the decrease of $\mathrm{W}, \mathrm{Mo}, \mathrm{Cr}$, $\mathrm{V}$. The increasing content of coarse carbides and $\mathrm{C}$ in the matrix increased the brittleness of the bimetallic transition zone and accelerated the formation of microcracks; hence, tensile fracture occurred in the bimetallic transition zone.

Therefore, an effective control of the chemical composition and microstructure in the bimetallic transition zone is quite necessary during the fabrication process of an HSS/
DCI composite roll. Due to the high content of $\mathrm{C}$ in the DCI core and the large contents of $\mathrm{W}, \mathrm{Mo}, \mathrm{Cr}, \mathrm{V}$ in the HSS layer, the formation of brittle carbides is inevitable. Hence, the mechanical properties of an HSS composite roll can be enhanced by reducing the elemental migration, designing an interlayer between the bimetals or using a roll core of forged or cast steel instead of the cast iron.

\section{Acknowledgements}

The authors are very grateful to the financial support of National Natural Science Foundation of China (52004188 and 51874084) and the China Postdoctoral Science Foundation (2019M652720). We also would like to thank the Analytical \& Testing Center of Wuhan University of Science and Technology for the help on EPMA analysis.

\section{REFERENCES}

1) Y. Sano, T. Hattori and M. Haga: ISIJ Int., 32 (1992), 1194.

2) M. Hashimoto, T. Tanaka, T. Inoue, M. Yamashita, R. Kurahashi and R. Terakado: ISIJ Int., 42 (2002), 982.

3) G. J. Li and M. J. Feng: J. Cent. South Univ., 21 (2014), 849.

4) C. K. Kim, J. I. Park, S. Lee, Y. C. Kim, N. J. Kim and J. S. Yang: Metall. Mater. Trans. A, 36 (2005), 87.

5) Y. K. Luan, N. N. Song, Y. L. Bai, X. H. Kang and D. Z. Li: J. Mater. Process. Technol., 210 (2010), 536.

6) H. G. Fu, A. M. Zhao, J. D. Xing and D. M. Fu: J. Iron Steel Res. Int., 9 (2002), 32.

7) A. Ludwig, A. Kharicha and M. H. Wu: Metall. Mater. Trans. B, 45 (2014), 36.

8) L. B. Medovar, A. K. Tsykulenko, V. Y. Saenko, A. V. Chernets, B. B. Fedorovskii, V. I. Us and I. A. Lantsman: Proc. Medovar Memorial Symp., Elmet-Roll Medovar Group Company, Kyiv, (2001), 49.

9) G. Polishko, G. Stovpchenko, L. Medovar and L. Kamkina: Ironmaking Steelmaking, 46 (2019), 789.

10) Y. L. Cao, Z. H. Jiang, Y. W. Dong, X. Deng, L. Medovar and G. Stovpchenko: ISIJ Int., 58 (2018), 1052.

11) Y. L. Cao, Z. H. Jiang, Y. W. Dong, X. Deng, L. Medovar and G. Stovpchenko: Metals, 8 (2018), 390.

12) Y. L. Cao, Z. H. Jiang, Y. W. Dong, G. Q. Li, Z. W. Hou and Q. Wang: Ironmaking Steelmaking, 47 (2020), 686.

13) Y. L. Cao, G. Q. Li, Z. H. Jiang, Y. W. Dong, Z. R. Zhao and C. R. Niu: High Temp. Mater. Process., 39 (2020), 270.

14) J. W. Park, H. C. Lee and S. Lee: Metall. Mater. Trans. A, 30 (1999), 399.

15) Z. X. Xiao, H. P. Li, J. H. Feng, Y. W. Mi, Y. H. Nie and F. X. Yin: J. Iron Steel Res., 30 (2018), 529 (in Chinese).

16) X. F. Zhou, F. Fang, F. Li and J. Q. Jiang: J. Mater. Sci., 46 (2011), 1196.

17) X. F. Zhou, F. Fang and J. J. Jiang: China Foundry, 8 (2011), 290.

18) M. Boccalini, Y. Matsubara and H. Goldenstein: AFS Trans., 104 (1996), 907.

19) F. X. Yin, Y. Liang, Z. X. Xiao, J. H. Feng, Z. B. Xie and Y. W. Mi: China Foundry, 16 (2019), 126.

20) China National Standard GB/T 6394-2002: 2003, Metal-methods for estimating the average grain size.

21) V. K. Afanasev, S. A. Storozhev, M. V. Maslyaev and V. N. Tolstoguzov: Steel Transl., 35 (2005), 76.

22) K. C. Hwang, S. Lee and H. C. Lee: Mater. Sci. Eng. A, 254 (1998), 282.

23) M. Boccalini and H. Goldenstein: Int. Mater. Rev., 46 (2001), 92.

24) H. Fredriksson, M. Hillert and M. Nica: Scand. J. Metall., 8 (1979), 115.

25) X. F. Zhou, F. Fang, J. Q. Jiang, W. L. Zhu and H. X. Xu: Mater. Sci. Technol., 28 (2012), 1499.

26) F. S. Pan, W. Q. Wang, A. T. Tang, L. Z. Wu, T. T. Liu and R. J. Cheng: Prog. Nat. Sci.: Mater. Int., 21 (2011), 180.

27) X. F. Zhou, F. Fang, G. Li and J. Q. Jiang: ISIJ Int., 50 (2010), 1151. 